\title{
Intoxicação experimental pelas favas de Stryphnodendron obovatum (Leg. Mimosoideae) em bovinos. 1. Caracterização do quadro clínico ${ }^{1}$
}

\author{
Marilene F. Brito ${ }^{2}$, Carlos H. Tokarnia ${ }^{3}$, Paulo V. Peixoto ${ }^{3}$, Heloisa K. Silva ${ }^{4}$ e Miriam \\ Nogueira ${ }^{5}$
}

\begin{abstract}
Brito M.F., Tokarnia C.H., Peixoto P.V., Silva H.K. \& Nogueira M. 2001. [Experimental poisoning by the burs of Stryphnodendron obovatum (Leg. Mimosoideae) in cattle. 1. Clinical aspects] Intoxicação experimental pelas favas de Stryphnodendron obovatum (Leg. Mimosoideae) em bovinos. 1. Caracterização do quadro clínico. Pesquisa Veterinária Brasileira 21(1):9-17. Projeto Sanidade Animal Embrapa/UFRRJ, Km 47, Seropédica, RJ 23851-970, Brazil.

In order to contribute to the characterization of the clinical-pathological picture of poisoning by Stryphnodendron obovatum Benth. (Leg. Mimosoideae), the pods of the tree were given orally to 17 young bovines. Six animals received the pods only once: Those which received doses of 10 and $20 \mathrm{~g} / \mathrm{kg}$, did not show any symptoms of poisoning. Those which received 30,40 and $40 \mathrm{~g} / \mathrm{kg}$ of pods, showed respectively moderate, severe and slight symptoms of poisoning, but all recovered. The animal that was fed $60 \mathrm{~g} / \mathrm{kg}$, died. Eleven bovines received repeated doses of the pods: Two of them, which received $2,5 \mathrm{~g} / \mathrm{kg}$ during 30 days, showed discrete to slight symptoms. Two animals received $5 \mathrm{~g} / \mathrm{kg}$ during 13 and 14 days and showed moderate to severe poisoning. Three animals received $10 \mathrm{~g} / \mathrm{kg}$ during 20,8 and 6 days; the first of them showed slight symptoms, the second died and the third showed moderate to severe symptoms. Two animals received $20 \mathrm{~g} / \mathrm{kg}$ during 2 and 3 days; the first showed slight symptoms, the second died. The two bovines which were fed 30 and $40 \mathrm{~g} / \mathrm{kg}$ during 2 days, died. First symptoms of poisoning were observed from the first day of the experiments on, and the clinical course varied from 3 to 63 days. Digestive disorders predominated in the clinical-pathological picture. Symptoms were mainly loss of appetite up to anorexia, faeces slightly dry to liquid, distention of the abdomen without tympanism, loss of ruminal fluid during rumination, decrease in ruminal activity up to atonia, ruminal acidosis, gastro-intestinal colics, sialorrhoea, apathy, loss of weight, debility, erosions and ulcers of the oral mucosa. There was congestion of the visible mucous membranes (without icterus) and of the interdigital parts of the hoofs, areas of focal alopecia and/or hypotrichia in the axillary regions, the lateral parts of the tighs and members, loss of hair on the tip of the tail, alterations which can be interpreted as slight photosensitization. In two bovines aspiration pneumonia was verified. In three animals intermittent prolapse of the prepuce was seen, as well as polacuria; sometimes these animals sighed during urination. In all experimental animals the urine was generally turbid, acid, dark ambar, with a sweat smell and high density, and there was proteinuria; in a few animals glicosuria was diagnosed. Bilirubine values were normal. Thus, the severe photosensitization reported in the literature could not be reproduced. Loss of weight, slight photosensitization and absence of icterus, observed in our experiments, are in accordance with the symptoms reported by farmers of the Cerrado-regions (Savanna) where S. obovatum occurs.
\end{abstract}

INDEX TERMS: Poisonous plants, experimental plant poisoning, Stryphnodendron obovatum, cattle.

\footnotetext{
${ }^{1}$ Aceito para publicação em 22 de novembro de 2000.

Parte da Discertação do primeiro autor, apresentada ao Curso de Doutorado em Sanidade Animal da Universidade Federal Rural do Rio de Janeiro (UFRRJ).

${ }^{2}$ Depto Epidemiologia e Saúde Pública, Instituto de Veterinária, UFRRJ, Km 47, Seropédica, RJ 23851-970. E-mail: marilene@ufrrj.br
}

\footnotetext{
${ }^{3}$ Depto Nutrição e Pastagem, Instituto de Zootecnia, UFRRJ. E-mail: tokarnia@ufrrj.br

${ }^{4}$ Médica Veterinária.

${ }^{5}$ Depto Medicina Veterinária, Universidade Federal Rural de Pernambuco (UFRPE), Rua Dom Manoel de Medeiros s/nº, Dois Irmãos, Recife, PE 52171-900.
} 
RESUMO.- Com o objetivo de melhor caracterizar o quadro clínico na intoxicação experimental por Stryphnodendron obovatum Benth., procedeu-se a administração das favas, por via oral, a seis bovinos, em doses únicas e a 11 outros, em doses repetidas. Doses únicas de 10 e $20 \mathrm{~g} / \mathrm{kg}$ não provocaram sintomas. Doses únicas de 30 e $40 \mathrm{~g} / \mathrm{kg}$ provocaram quadros clínicos desde leves até graves, porém só morreu o animal que ingeriu $60 \mathrm{~g} / \mathrm{kg}$. Os animais que ingeriram doses repetidas de $2,5 \mathrm{~g} / \mathrm{kg}$ por 30 dias adoeceram levemente, já os que receberam $5 \mathrm{~g} / \mathrm{kg}$ durante 13 e 14 dias mostraram sintomatologia moderada e grave, respectivamente. Um bovino que recebeu $10 \mathrm{~g} / \mathrm{kg}$ por 8 dias, outro que ingeriu $20 \mathrm{~g} / \mathrm{kg}$ por 3 dias e dois outros que receberam 30 e $40 \mathrm{~g} / \mathrm{kg}$ por 2

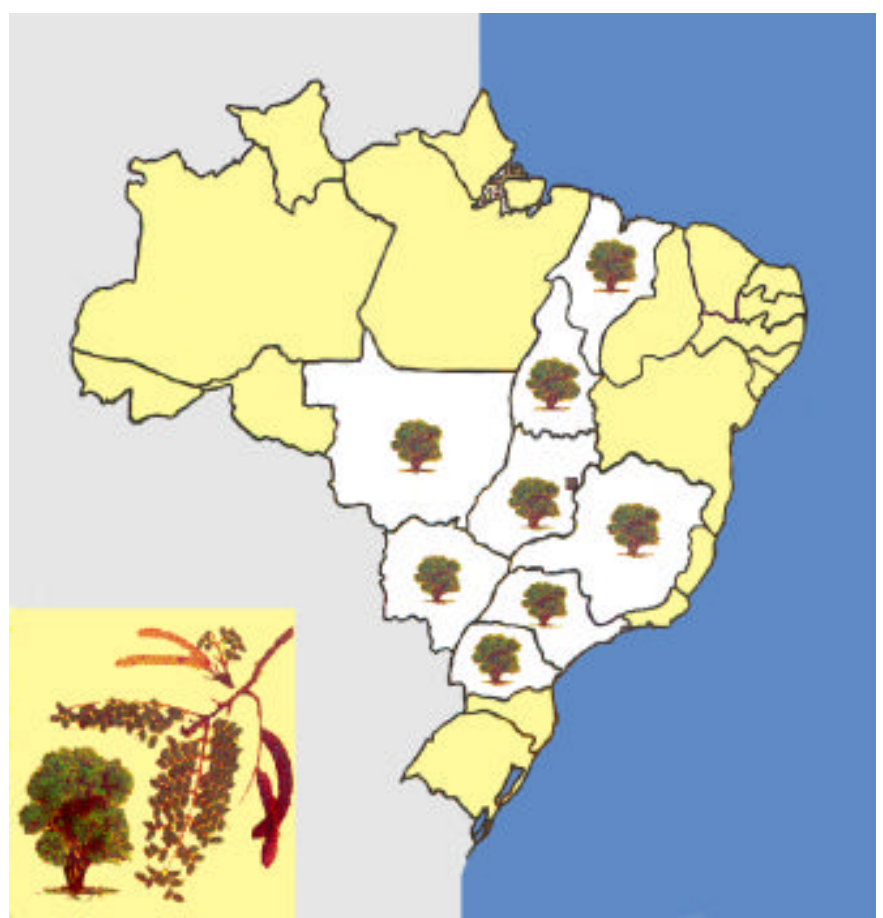

Fig. 1. Distribuição de Stryphnodendron obovatum no Brasil.

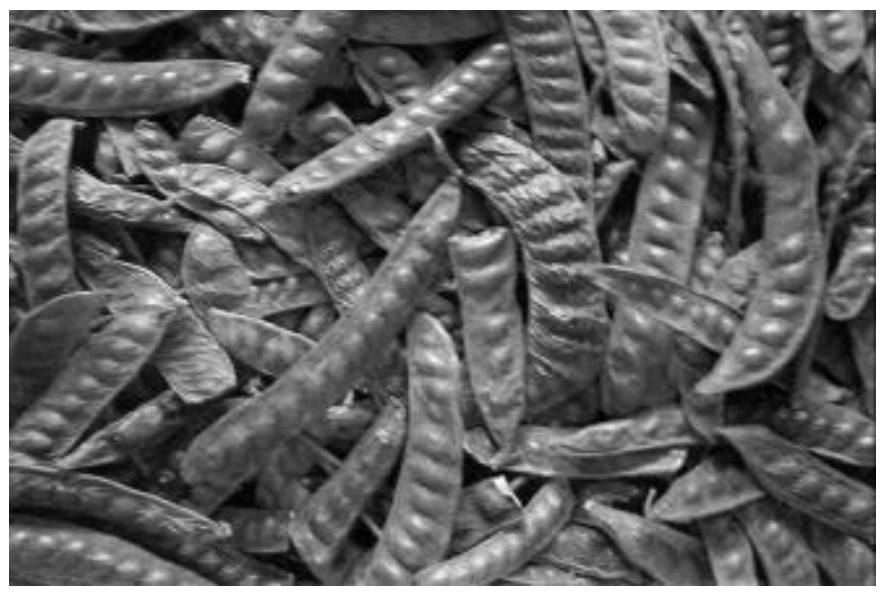

Fig. 2. Favas maduras de Stryphnodendron obovatum. dias morreram da intoxicação. Os outros três bovinos que receberam doses repetidas (dois com doses $\mathrm{de} 10 \mathrm{~g} / \mathrm{kg}$ por 20 e 6 dias, outro com dose de $20 \mathrm{~g} / \mathrm{kg}$ por 2 dias) adoeceram, mas se recuperaram. Os primeiros sintomas de intoxicação, em ambos os grupos, foram observados a partir do primeiro dia do experimento e a evolução variou de 3 a 63 dias. Os sintomas consistiram em anorexia, fezes levemente ressecadas a líquidas, distensão do abdômen, sem timpanismo, perda de fluido ruminal durante a ruminação, atonia e acidose ruminal, cólica, sialorréia, apatia, emagrecimento, fraqueza, erosões e úlceras na cavidade oral. Em alguns animais foram observadas congestão de mucosas visíveis (sem icterícia) e de partes despigmentadas da pele, do córion laminar e da região interdigital dos quatro membros, áreas de alopecia focal e/ou hipotricose nas regiões axilares, na face lateral das coxas e membros e perda dos pêlos da ponta da cauda, alterações estas que podem ser interpretadas como as de leve fotossensibilização. Em três bovinos se observaram relaxamento intermitente do prepúcio, miç̧ão freqüente e em gotejamento e, por vezes, gemidos ao eliminar a urina que, em geral, se apresentava turva, ácida, de cor âmbar-escura, com odor adocicado e com a densidade elevada; os níveis de bilirrubina na urina foram normais.

TERMOS DE INDEXAÇÃO: Plantas tóxicas, intoxicação experimental por plantas, Stryphnodendron obovatum, bovinos.

\section{INTRODUÇÃO}

O gênero Stryphnodendron comporta 32 taxa (variedades) e é tipicamente brasileiro (Occhioni 1990). Nesse gênero encontram-se algumas espécies tóxicas de interesse pecuário, dentre as quais inclui-se Stryphnodendron obovatum Benth., da família Leguminosae Mimosoideae, que é uma árvore pequena, conhecida popularmente como "barbatimão" ou "barbatimão de folha miúda". Essa planta tem ampla distribuição nos campos, cerrados e cerradões das regiões Centro-Oeste e Sudeste do Brasil (Fig. 1), estendendo-se principalmente pelos Estados de Mato Grosso, Mato Grosso do Sul, Goiás e São Paulo (Andrade \& Mattos 1976, Occhioni, 1990) e suas favas (Fig. 2) são largamente consumidas pelo gado, que é criado

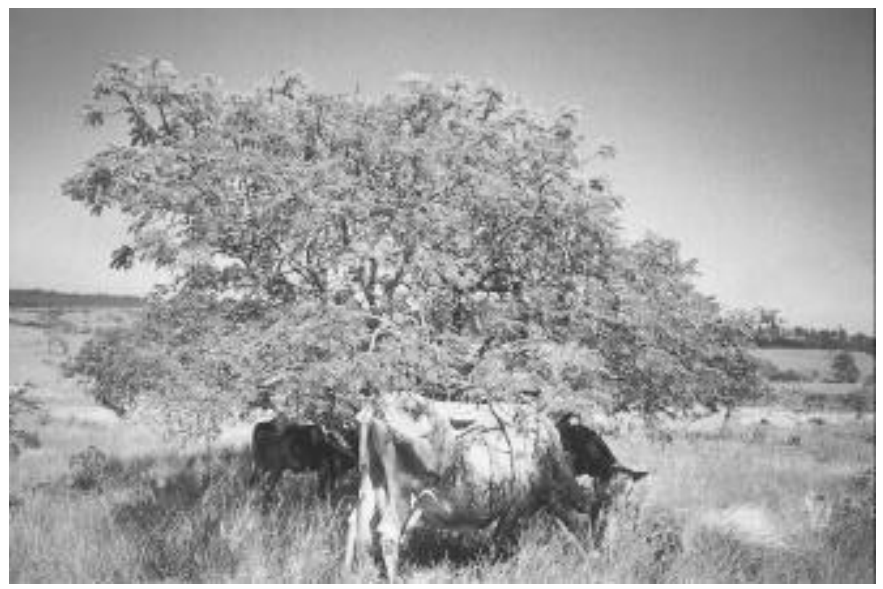

Fig. 3. Bovinos se alimentando das favas de Stryphnodendron obovatum; região de Cerrado, Botucatu, SP. 
extensiva ou semi-extensivamente naquelas áreas. A época de frutificação coincide com o período de seca e escassez de pastagem. Os bovinos se alimentam das favas maduras ainda na árvore ou quando as mesmas caem ao solo (Fig. 3). Em viagens aos Estados de Mato Grosso e São Paulo, em regiões de ocorrência de $S$. obovatum, verificamos que os bovinos comem avidamente as favas, mas não ingerem as folhas. Segundo históricos de criadores, os animais apreciam os frutos de S. obovatum e alguns até ficam viciados em comê-los, não engordam, muitos até emagrecem, ficam debilitados e acabam abortando.

Há controvérsias sobre a toxidez das favas de S. obovatum na literatura. Camargo (1965) incrimina S. obovatum como causa de intoxicação natural em bovinos com quadro de fotossensibilização, por vezes associado a icterícia, erosões na mucosa oral, sialorréia, constipação e em alguns casos, prostração e morte. Nos estudos experimentais, esse pesquisador administrou as favas a cinco bovinos, dois dos quais apresentaram lesões de fotossensibilização, além de icterícia, apatia e anorexia. Porém, Tokarnia (dados não-publicados, 1960 a 1983) administrou as favas de S. obovatum a dez bovinos, em doses variadas e repetidas, sem conseguir demonstrar a sua ação fotossensibilizante; os únicos sintomas que observou foram anorexia e diarréia. Mais tarde Tokarnia et al. (1998) confirmaram experimentalmente a ação abortiva das favas desta planta, porém novamente não conseguiram reproduzir a fotossensibilização.

É pertinente observar que existem duas outras espécies tóxicas de Stryphnodendron, que são Stryphnodendron coriaceum Benth. (Döbereiner \& Canella 1956, Tokarnia et al. 1991) e Stryphnodendron barbatimao Mart. (Pereira et al. 1989a). A toxidez de S. coriaceum foi demonstrada por Döbereiner \& Canella (1956). Esta árvore é conhecida popularmente como "barbartimão", "barbartimão do Nordeste" ou "barbatimão do Piauí"; ela é uma importante planta tóxica da região Nordeste, especialmente do Piauí, Maranhão, Oeste da Bahia e do Norte de Goiás. Na espécie bovina, Tokarnia et al. (1991) revelaram que 12 , dos 21 bovinos que receberam as favas, sofreram intoxicação grave e nove destes morreram; o quadro predominante foi digestivo, apresentando, principalmente, congestão da mucosa oral, sialorréia, regurgitamento do conteúdo ruminal, broncopneumonia por aspiração, andar cambaleante, diarréia, perda dos pêlos da ponta da cauda, fotossensibilização e icterícia leves.

A toxidez de S. barbatimao foi demonstrada para bovinos por Pereira et al. (1989a,b,c) que, no entanto, não fornecem dados sobre a ocorrência da intoxicação natural. Esta planta, ocorrente em cerrados do Nordeste e Sudeste do Brasil, é conhecida vulgarmente como "barbatimão", "faveiro" ou "enche-cangalha". Pereira et al. (1989a), observaram como principais sintomas apatia, anorexia, sialorréia, desidratação, fezes fétidas, de consistência variável e com muco, incoordenação, temperamento linfático, anemia, lacrimejamento, polaciúria, ranger de dentes, dor abdominal, perda de peso, queda dos pêlos do dorso, do pescoço e de áreas sombreadas e depois de todo corpo, coroa dos cascos avermelhadas e edemaciadas, mucosas hiperêmicas e em alguns, fotossensibilização. Pereira et al. (1989c) destacam a acidez e diminuição da densidade na urina nos bovinos intoxicados por $S$. barbatimao.

Este estudo consistiu na administração das favas de Stryphnodendron obovatum a bovinos, em diversas dosagens, com o fim de caracterizar o quadro clínico desta intoxicação.

\section{MATERIAL E MÉTODOS}

Os experimentos e exames laboratoriais foram realizados nas instalações do Convênio Projeto Sanidade Animal da Empresa Brasileira de Pesquisa Agropecuária (Embrapa) / Universidade Federal Rural do Rio de Janeiro (UFRRJ), Seropédica, RJ. Foram utilizados 20 bovinos, sem raça definida, de ambos os sexos, com pesos entre $85 \mathrm{~kg}$ e $255 \mathrm{~kg}$ e com idades entre 1 e 3 anos, três dos quais foram utilizados como controles. Trinta dias antes do início dos trabalhos experimentais os animais foram colocados em baias individuais, visando a adaptação e observação clínica prévia. Durante o período de adaptação e por toda a fase experimental, os animais foram submetidos a exames clínicos pelo menos duas vezes ao dia. Pela manhã, recebiam ração balanceada ${ }^{6}$ e mistura mineral ${ }^{7}$, posteriormente eram levados ao solário e, ao retornarem, recebiam capimelefante (Pennisetum purpureum) e capim-angola (Brachiaria mutica). À tarde voltavam ao solário e ao retornarem recebiam o mesmo tipo de forragem. As favas maduras de Stryphnodendron obovatum foram coletadas nos municípios de Botucatu, SP e Santo Antonio do Leverger, MT, em 1993, e em Ribas do Rio Pardo, MS, em 1998, diretamente das árvores e do solo onde os bovinos pastavam (Fig. 2). Os experimentos foram realizados em duas etapas: a primeira de novembro de 1993 a fevereiro de 1994 e a segunda de novembro de 1998 a janeiro de 1999 . As favas de $S$. obovatum, moídas em moinho martelo e levemente umedecidas, foram administradas aos bovinos, por via oral, em doses únicas ou repetidas durante 1 a 30 dias; nos casos de doses repetidas, o número de administrações era condicionado ao aparecimento de anorexia. Nos casos em que não houve anorexia, administrou-se a planta por, no máximo, 30 dias.

Todos os bovinos eram avaliados quanto ao seu estado geral, comportamento, atitude, apetite, cor das mucosas, exame físico das fezes, aparência da pele, temperatura retal, frequiências cardíaca e respiratória e atividade do rúmen. A urinálise foi realizada com o auxílio de fitas reativas ${ }^{8}$.

\section{Exames do fluido ruminal}

Na primeira série de experimentos, foram realizadas três avaliações do fluido ruminal dos bovinos experimentais. A primeira, antes da administração das favas. A segunda, 24 horas após a administração da última dose e a terceira, após os animais apresentarem-se clinicamente sadios. Nos bovinos-controle, foram realizadas duas coletas; uma no início e a outra no final do período experimental. Na segunda série de experimentos, os exames de fluido ruminal foram realizados diariamente. As coletas foram feitas através de sonda esofágica de plástico flexível e o fluido ruminal era analisado imediatamente após a coleta. Nas duas séries, foram avaliados: cor, odor, aspecto, flotação-sedimentação, pH, redução do azul de metileno, acidez total e protozoários.

A cor, o odor e o aspecto foram avaliados de forma subjetiva; a flotação-sedimentação foi realizada segundo Nichols \& Penn (1958); a concentração de íons hidrogênio foi mensurada através do papel indicador ${ }^{9}$, a prova do azul de metileno foi realizada de acordo com

\footnotetext{
${ }^{6}$ Novilhina-C

${ }^{7}$ Suplemento Mineral 65

${ }^{8}$ Multistix 10 SG, Bayer

${ }^{9}$ Fitas indicadoras de $\mathrm{pH}$, Merck
} 
Dirksen (1969); a prova da acidez total titulável foi realizada segundo a técnica de Jonov, citada por Slanina \& Rossow (1964). Os protozoários foram avaliados através de microscopia direta, segundo Baumgartner (1983), observando-se densidade, motilidade, relação vivos / mortos e predominância.

\section{RESULTADOS}

Dos seis bovinos que receberam as favas em doses únicas, os de $10 \mathrm{~g} / \mathrm{kg}$ e $20 \mathrm{~g} / \mathrm{kg}$ não adoeceram; os três de $30 \mathrm{~g} / \mathrm{kg}, 40 \mathrm{~g} /$ $\mathrm{kg}$ e $40 \mathrm{~g} / \mathrm{kg}$ adoeceram com quadros moderado, grave e leve, respectivamente, e todos se recuperaram. $O$ bovino que recebeu $60 \mathrm{~g} / \mathrm{kg}$, morreu.

Dos 11 bovinos que receberam as favas em doses repetidas, dois receberam $2,5 \mathrm{~g} / \mathrm{kg}$ durante 30 dias; dois receberam $5 \mathrm{~g} / \mathrm{kg}$ durante 13 e 14 dias; três receberam $10 \mathrm{~g} / \mathrm{kg}$ durante 20,8 e 6 dias; dois receberam $20 \mathrm{~g} / \mathrm{kg}$ durante 2 e 3 dias; um recebeu $30 \mathrm{~g} / \mathrm{kg}$ durante 2 dias e o último bovino recebeu $40 \mathrm{~g} / \mathrm{kg}$ durante 2 dias. Desses 11 bovinos que receberam doses repetidas, um adoeceu discretamente, três adoeceram levemente, sete adoeceram gravemente e destes, quatro morreram. Dos que receberam doses únicas, os primeiros sintomas foram observados no primeiro e segundo dias do experimento (DE) e a evolução variou de 3 a 63 dias e naqueles que receberam doses repetidas, os primeiros sintomas foram observados do primeiro ao quarto DE e a evolução variou de 3 a 50 dias. (Quadro 1)

Dentre os sintomas digestivos (Quadros 2 e 3) observaramse diminuição progressiva do apetite até anorexia, odor das favas nas fezes; as fezes mostravam-se levemente ressecadas, marrom-escuras, por vezes fétidas, em alguns casos sob forma de discos agrupados e com muco tingido de sangue, em ou- tros, tinham consistência semilíquida até líquida, em grande quantidade, por vezes, sob forma de jatos e de cor amarelada.

Distensão do abdômen (Fig. 4), sem timpanismo, foi observada nos casos em que os bovinos ingeriram doses superiores a $30 \mathrm{~g} / \mathrm{kg}$ das favas. Perda de fluido ruminal durante a ruminação (Fig. 5 ) foi observada nos casos em que a evolução do quadro clínico ultrapassou o $13^{\circ} \mathrm{DE}$. Diminuição da atividade ruminal até atonia do rúmen ocorreu tanto nos bovinos que ingeriram doses únicas, quanto nos que ingeriram doses repetidas das favas. Nos dez bovinos que apresentaram quadros clínicos de moderado a grave, verificou-se que houve acidose ruminal também de moderada a grave, culminando com êxito letal em cinco deles. As principais modificações observadas no fluido ruminal foram alteração da cor para castanho-amarelado, castanho-acinzentado, leitosoacinzentado, até castanho-oliva. A acidez do fluido ruminal atingiu valores mínimos de até 4,5 no $1^{\circ}$ e $2^{\circ}$ DE ou nos primeiros dois dias após o término da ingestão das favas. $O$ odor variou de discretamente ácido para ácido-penetrante e até pútrido. $\mathrm{O}$ aspecto era aquoso e, por vezes, espumoso, com sedimento grosseiro quando se podia visualizar pequenos fragmentos das favas. Houve diminuição até ausência da atividade de redução do azul de metileno, por vezes, com formação do anel pouco definido. A acidez total titulável elevou-se em níveis de até 72 unidades clínicas (UC). A prova de sedimentação e flutuação, em alguns casos, teve seu tempo abreviado. Os protozoários diminuíram progressivamente em densidade e motilidade e nos casos mais graves já se apresentavam $100 \%$ mortos a partir do $1^{\circ}$ DE ou no primeiro dia após a última ingestão das favas.

Alguns bovinos apresentaram cólica, manifestada através

Quadro 1. Intoxicação experimental pelas favas de Stryphnodendron obovatum em bovinos. Dados gerais

\begin{tabular}{|c|c|c|c|c|c|c|c|c|c|c|}
\hline \multicolumn{2}{|c|}{ Bovino } & \multirow{2}{*}{$\begin{array}{c}\text { Período de } \\
\text { administração }\end{array}$} & \multirow{2}{*}{$\begin{array}{l}\text { Dose } \\
\text { diária } \\
(\mathrm{g} / \mathrm{kg})\end{array}$} & \multirow{2}{*}{$\begin{array}{l}\text { Dose } \\
\text { diária } \\
\text { (g) }\end{array}$} & \multirow{2}{*}{$\begin{array}{l}\text { Número de } \\
\text { dias de } \\
\text { ingestão }\end{array}$} & \multirow{2}{*}{$\begin{array}{l}\text { Dose } \\
\text { total } \\
(\mathrm{g} / \mathrm{kg})\end{array}$} & \multirow{2}{*}{$\begin{array}{c}\text { Total } \\
\text { ingerido } \\
\text { (g) }\end{array}$} & \multirow{2}{*}{$\begin{array}{c}\text { Início dos } \\
\text { sintomas } \\
\text { após a } 1^{\mathrm{a}} \\
\text { administração }\end{array}$} & \multirow{2}{*}{$\begin{array}{l}\text { Intensidade } \\
\text { dos sintomas } \\
\text { e desfecho }\end{array}$} & \multirow[t]{2}{*}{ Evolução } \\
\hline $\mathrm{N}^{\mathbf{o}}$ & $\begin{array}{r}\text { Peso } \\
(\mathrm{kg})\end{array}$ & & & & & & & & & \\
\hline 5213 & 132 & -* & controle & - & - & - & - & - & - & - \\
\hline 5211 & 119 & - & controle & - & - & - & - & - & - & - \\
\hline 181 & 118 & - & controle & - & - & - & - & - & - & - \\
\hline 860 & 110 & $15 / 12 / 98$ & 10 & 1.100 & 1 & 10 & 1.100 & - & Não adoeceu & - \\
\hline 801 & 164 & $15 / 12 / 98$ & 20 & 3.280 & 1 & 20 & 3.280 & - & Não adoeceu & - \\
\hline $181 \mathrm{~A}$ & 114 & $07 / 11 / 98$ & 30 & 3.420 & 1 & 30 & 3.420 & $2^{\mathrm{o}}$ dia & Adoeceu ++ & 31 dias \\
\hline 866 & 110 & $17 / 11 / 98$ & 40 & 4.400 & 1 & 40 & 4.400 & $1^{\circ} \mathrm{dia}$ & Adoeceu +++ & 63 dias \\
\hline 5214 & 179 & 29/12/93 & 40 & 7.160 & 1 & 40 & 7.160 & $1^{\mathrm{o}} \mathrm{dia}$ & Adoeceu + & 4 dias \\
\hline 840 & 117 & $23 / 11 / 98$ & 60 & 7.020 & 1 & 60 & 7.020 & $1^{o}$ dia & Morreu & 4 dias \\
\hline $5211 \mathrm{~A}$ & 129 & 09/12/93 a $07 / 01 / 94$ & 2,5 & 322 & 30 & 75 & 9.660 & $4^{\mathrm{o}}$ dia & Adoeceu (+) & 12 dias \\
\hline 5212 & 85 & $09 / 12 / 93$ a $07 / 01 / 94$ & 2,5 & 212,5 & 30 & 75 & 6.375 & $3^{\circ}$ dia & Adoeceu + & 26 dias \\
\hline 5202 & 211 & $28 / 11 / 93$ a $10 / 12 / 93$ & 5,0 & 1.055 & 13 & 65 & 13.715 & $1^{\circ}$ dia & Adoeceu +++ & 36 dias \\
\hline 5206 & 173 & $25 / 11 / 93$ a $08 / 12 / 93$ & 5,0 & 865 & 14 & 70 & 12.110 & $3^{\circ}$ dia & Adoeceu $++/+++$ & 23 dias \\
\hline 5204 & 122 & $25 / 11 / 93$ a $14 / 12 / 93$ & 10 & 1.220 & 20 & 200 & 24.400 & $3^{\circ}$ dia & Adoeceu + & 28 dias \\
\hline 5210 & 130 & $30 / 11 / 93$ a $07 / 12 / 93$ & 10 & 1.300 & 8 & 80 & 10.400 & $1^{\circ}$ dia & Morreu & 13 dias \\
\hline 180 & 145 & $23 / 11 / 98$ a $28 / 11 / 98$ & 10 & 1.450 & 6 & 60 & 8.700 & $1^{\circ}$ dia & Adoeceu $++/+++$ & 50 dias \\
\hline 5209 & 86 & $09 / 12 / 93$ a $10 / 12 / 93$ & 20 & 1.720 & 2 & 40 & 3.440 & $2^{o}$ dia & Adoeceu + & 13 dias \\
\hline 4919 & 255 & $25 / 11 / 93$ a $27 / 11 / 93$ & 20 & 5.100 & 3 & 60 & 15.300 & $2^{\circ}$ dia & Morreu & 6 dias \\
\hline 802 & 161 & $10 / 11 / 98$ a $11 / 11 / 98$ & 30 & 4.830 & 2 & 60 & 9.660 & $1^{o}$ dia & Morreu & 6 dias \\
\hline 182 & 176 & $06 / 11 / 98$ a $07 / 11 / 98$ & 40 & 7.040 & 2 & 80 & 14.080 & $2^{o}$ dia & Morreu & 3 dias \\
\hline
\end{tabular}

*- $=$ Sintomas ausentes, $+=$ leves, $++=$ moderados, $+++=$ graves. 
Quadro 2. Sintomas na intoxicação experimental pelas favas de Stryphnodendron obovatum nos bovinos que ingeriram doses únicas

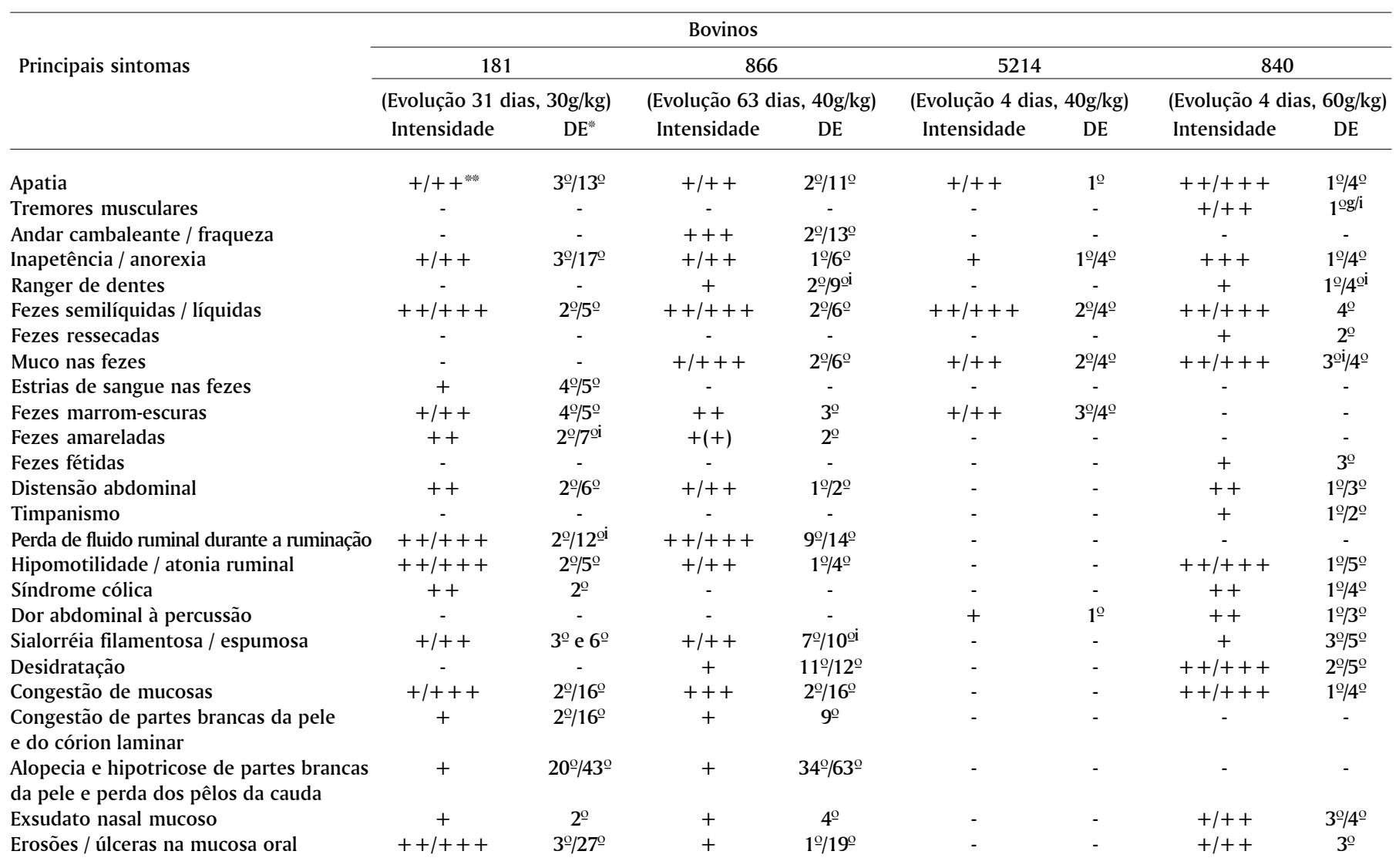

${ }^{*} \mathrm{DE}=\operatorname{dia}(\mathbf{s})$ do experimento.

***- $=$ Sintomas ausentes, $+=$ leves, $++=$ moderados, $+++=$ graves, $\mathrm{i}=$ intermitente.

de sinais de inquietação, deitar-se e levantar-se com freqüência e cuidado, dor abdominal à percussão, dorso encurvado, olhar para o flanco, escoicear o ventre e levantar os membros anteriores e posteriores, geralmente do $1^{\circ}$ ao $13^{\circ} \mathrm{DE}$. Também foram observados sialorréia filamentosa e/ou espumosa e ranger de dentes.

Dentre os sinais não relacionados ao tubo digestivo, observaram-se apatia, tendência ao decúbito esterno-abdominal, sinais de fraqueza como andar cambaleante, trôpego, às vezes trocando os membros, tremores musculares generalizados, sinais de desidratação, erosões e úlceras na mucosa da cavidade oral (Fig. 6), congestão de mucosas visíveis (sem icterícia) e de partes despigmentadas da pele, acompanhados de áreas de alopecia focal e/ou hipotricose, geralmente bilateral, na região axilar (Fig. 7), na face lateral das coxas e na extremidade distal dos membros anteriores e posteriores, principalmente nas quartelas, no córion laminar e na região interdigital (Fig. 8). Também se observou perda dos pêlos da ponta da cauda em dois bovinos os quais tiveram uma evolução prolongada. Em dois bovinos, observou-se pneumonia por aspiração, em outros, foi notada a presença de exsudato nasal mucoso a muco-purulento, bilateral e narinas ressecadas. Não houve alterações significativas na temperatura corporal ou nas freqüências cardíaca e respiratória.
Dentre as alterações urinárias, observaram-se, em dois bovinos que ingeriram dose única e doses repetidas, relaxamento intermitente do prepúcio, micção freqüente e em gotejamento e, por vezes, gemidos ao urinar. A urinálise revelou que a urina tornava-se escura, variando do âmbar-claro ao marrom-escuro, com odor adocicado, às vezes turva, ácida, com pH mínimo de 4,0, com a densidade elevada, por vezes atingindo valores superiores a 1.045. Proteinúria também foi verificada e, em média, com valores entre $30 \mathrm{mg} / \mathrm{dl}$ e $100 \mathrm{mg} /$ dl. Três bovinos apresentaram glicosúria de $30 \mathrm{mg} / \mathrm{dl}$ a $1.000 \mathrm{mg} / \mathrm{dl}$. Os níveis de bilirrubina na urina foram normais.

Houve emagrecimento e a perda de peso foi mais acentuada entre os animais que tiveram uma evolução mais longa; em geral acima de 12 dias, em ambos os grupos. Alguns tinham peso oscilante, perdiam e recuperavam peso sucessivamente (Quadro 4).

\section{DISCUSSÃO E CONCLUSÕES}

As manifestações da intoxicação por Stryphnodendron obovatum em bovinos foram bastante uniformes e se relacionaram, predominantemente, ao sistema digestivo. A dose letal foi de $60 \mathrm{~g} / \mathrm{kg}$ em dose única ou 60 a $80 \mathrm{~g} / \mathrm{kg}$ subdividida em 2 a 8 vezes.

Apesar da ingestão de consideráveis quantidades de fa- 


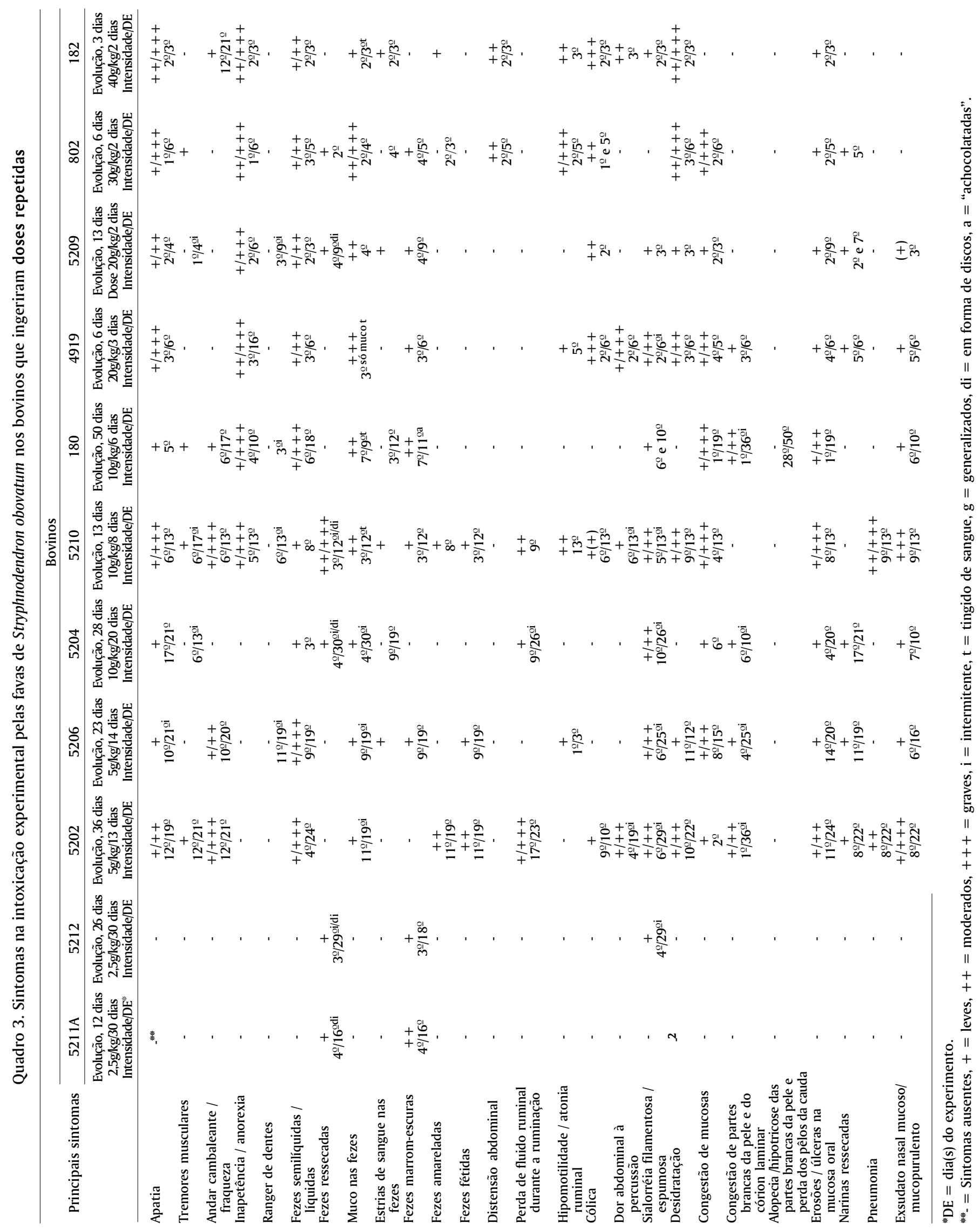




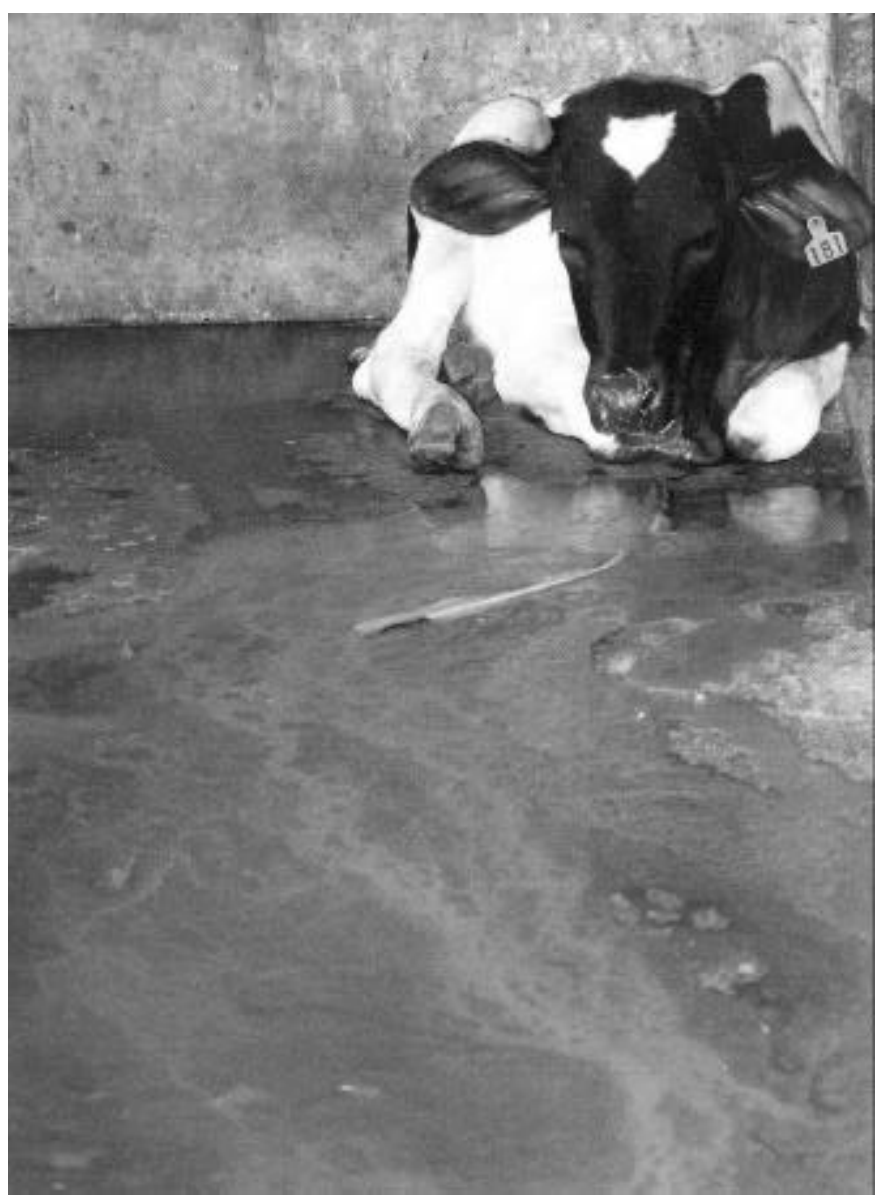

vas de $S$. obovatum, de diversas procedências, sete de 17 bovinos apresentaram apenas leves alterações na pele e nenhum deles apresentou icterícia. Não observamos um quadro grave de fotossensibilização, como o apresentado por Camargo (1965). Também nos experimentos realizados por Tokarnia (dados não-publicados, 1960 a 1983) em 14 bovinos e por Tokarnia et al. (1998) em 7 bovinos, nenhum deles desenvolveu sinais de fotossensibilização e icterícia. Porém, alguns dos bovinos intoxicados por S. obovatum, nesse estudo, apresentaram lesões de pele que podem ser interpretadas como as de leve fotossensibilização.

Verificou-se que o emagrecimento, a ausência de quadros graves de fotossensibilização e a ausência de icterícia são condizentes com os históricos colhidos nas regiões de ocorrência da planta, os quais também não fazem referências à fotossensibilização e à icterícia.

Observou-se ainda que os dez bovinos que tiveram quadro clínico moderado ou grave, evidenciaram acidose ruminal moderada a grave e cinco destes morreram. Acreditamos que a ingestão de doses elevadas das favas provocaram modificações na microbiota ruminal que culminaram com o quadro de acidose. $O$ desequilíbrio hidroeletrolítico e a desidratação, determinados pela diarréia e sialorréia marcadas, associados à acidose ruminal, provavelmente devem ter induzido à acidose metabólica e choque nos animais.

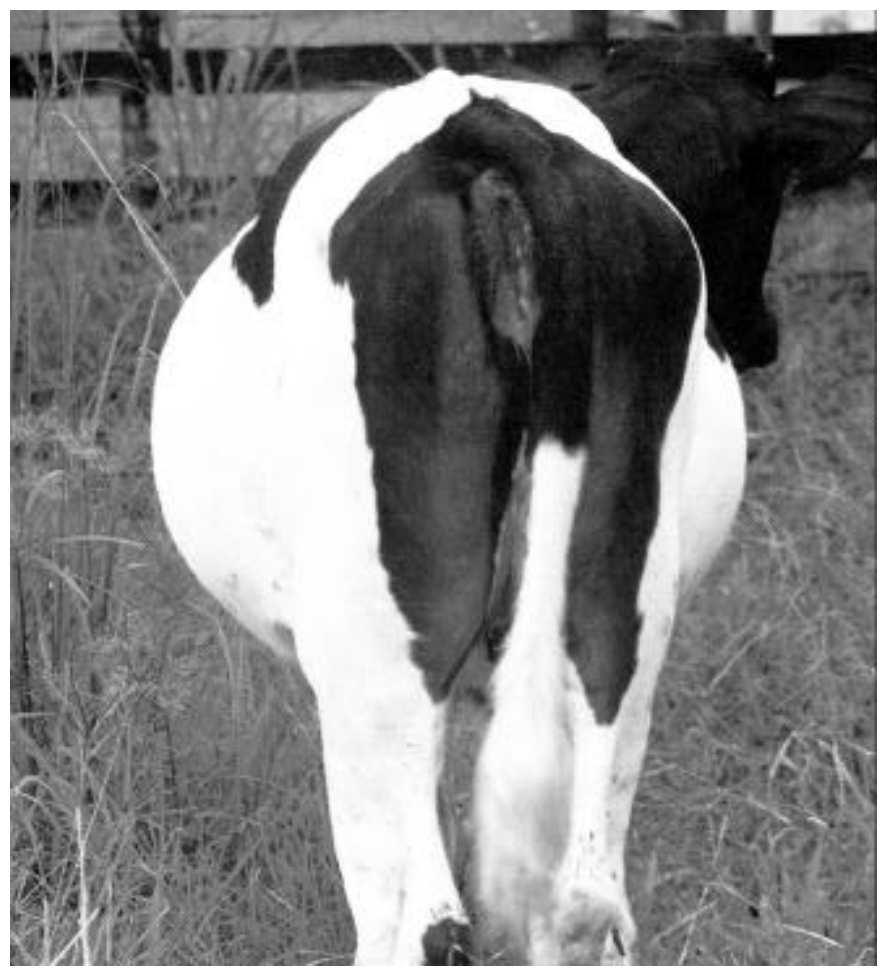

Fig. 4. Distensão abdominal (Bovino 181A), na intoxicação experimental pelas favas de Stryphnodendron obovatum.

Fig. 5. Perda de fluido ruminal, durante a ruminação (Bovino 181A), na intoxicação experimental pelas favas de Stryphnodendron obovatum.

Nos animais que ingeriram doses menores únicas ou nas administrações por períodos mais prolongados, os quadros clínicos foram mais suaves sem desenvolvimento de acidose ruminal, porém, a perda de fluido ruminal e o emagrecimento foram mais acentuados.

Nos bovinos intoxicados por $S$. coriaceum se observaram sintomas digestivos e adicionalmente leve icterícia e fotossensibilização (Döbereiner \& Canella 1956, Tokarnia et al. 1991, 2000).

Pereira et al. (1989a), em seus experimentos com Stryphnodendron barbatimao em bovinos, além dos sintomas digestivos, obtiveram alguns sinais de fotossensibilização que não foram encontrados no quadro causado por S. obovatum, como queda de pêlos no dorso e pescoço, com generalização da alopecia e desprendimento de partes ressecadas da pele, coroa dos cascos edemaciada e edemas submandibular e palpebral.

As modificações da urina observadas em nossos experimentos, tais como pH ácido e densidade, proteínas e glicose elevados, especialmente naqueles bovinos que desenvolveram quadro de acidose ruminal, estão de acordo com as descritas por Dirksen (1981). Nos exames laboratoriais, Pereira et al. (1989c) determinaram, a exemplo do que se observou na intoxicação por $S$. obovatum, acidez na urina, porém, ao contrário do encontrado em nosso estudo, a densi- 


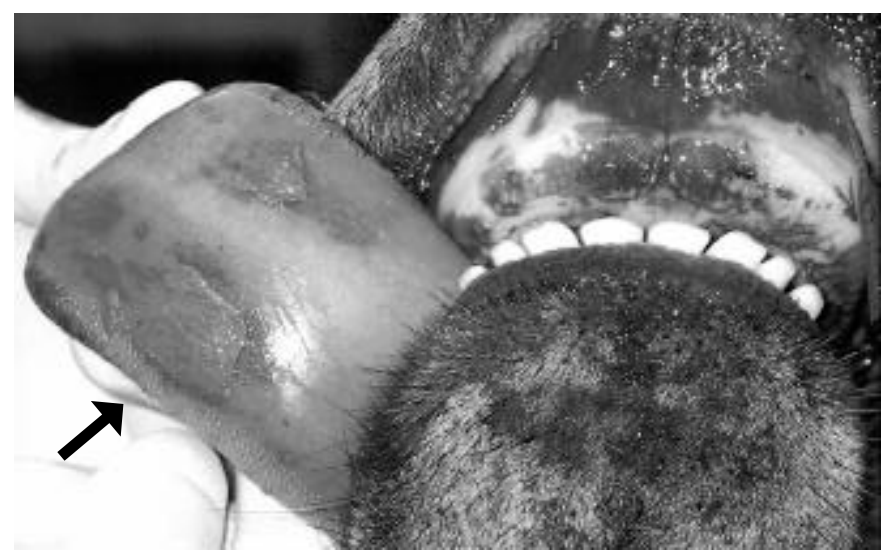

Fig. 6. Congestão e erosões/úlceras na face ventral da língua, algumas cobertas por material necrótico amarelado e deposição de pigmento castanho-amarelado no pulvino dental (Bovino 181A), na intoxicação experimental pelas favas de Stryphnodendron obovatum.

Fig. 7. Hiperemia e alopecia da região axilar (Bovino 181A), na intoxicação experimental pelas favas de Stryphnodendron obovatum.

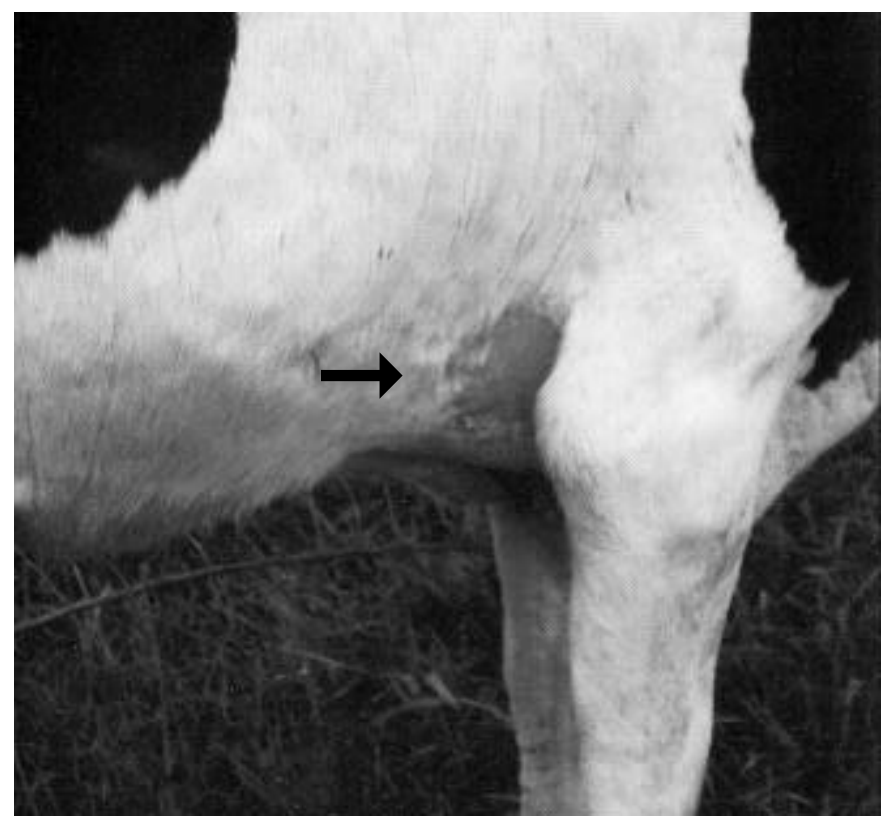

Quadro 4. Pesos $(\mathrm{kg})$ dos bovinos controle e dos bovinos que receberam doses únicas e doses repetidas na intoxicaçãoexperimental pelas favas de Stryphnodendron obovatum

\begin{tabular}{|c|c|c|c|c|c|c|c|c|c|c|c|c|c|c|c|c|c|c|c|c|}
\hline \multirow[t]{4}{*}{ Data } & \multicolumn{20}{|c|}{ Bovino } \\
\hline & \multicolumn{3}{|c|}{ Controles } & \multirow[t]{2}{*}{860} & \multirow{2}{*}{\multicolumn{2}{|c|}{$\begin{array}{l}801 \quad 181 \mathrm{~A} \\
\text { Doses únicas }\end{array}$}} & \multirow{2}{*}{\multicolumn{2}{|c|}{$\begin{array}{cc}866 & 5214 \\
(\mathrm{~g} / \mathrm{kg}) / \text { Peso }\end{array}$}} & \multirow[t]{2}{*}{840} & \multirow{2}{*}{\multicolumn{2}{|c|}{ 5211A 5212}} & 5202 & 5206 & 5204 & 5210 & \multirow{2}{*}{$\begin{array}{c}180 \\
\text { dias) } / \mathrm{Pe}\end{array}$} & \multirow{2}{*}{$\begin{array}{c}5209 \\
\text { so }\end{array}$} & \multirow[t]{2}{*}{4919} & \multirow[t]{2}{*}{802} & 182 \\
\hline & 5213 & 5211 & 181 & & & & & & & & & Do & ses rep & etidas & $(g / k g x$ & & & & & \\
\hline & Peso & Peso & Peso & 10 & 20 & 30 & 40 & 40 & 60 & $2,5 \times 30$ & $2,5 \times 30$ & $5 \times 13$ & $5 \times 14$ & $10 \times 20$ & $10 \times 8$ & $10 \times 6$ & $20 \times 2$ & $20 \times 3$ & $30 \times 2$ & $40 \times 2$ \\
\hline 21.11 .93 & & 119 & & & & & & & & & & & 165 & 122 & & & & 247 & & \\
\hline 26.11 .93 & & 120 & & & & & & & & & & & 177 & 117 & & & & 255 & & \\
\hline 28.11 .93 & & & & & & & & & & & & & & & 130 & & & & & \\
\hline 03.12 .93 & & 124 & & & & & & & & & & 213 & 169 & 118 & 132 & & 86 & & & \\
\hline 08.12 .93 & & & & & & & & & & & & & & & 114 & & & & & \\
\hline 09.12 .93 & & 129 & & & & & & & & 129 & 85 & & & & & & & & & \\
\hline 10.12 .93 & & & & & & & & & & & & 188 & 160 & 122 & 107 & & 88 & & & \\
\hline 18.12 .93 & & & & & & & & & & 124 & 80 & 182 & 158 & 113 & & & 87 & & & \\
\hline 24.12 .93 & 132 & & & & & & & & & 127 & 83 & 181 & 171 & 120 & & & 93 & & & \\
\hline 27.12 .93 & 132 & & & & & & & 182 & & & & & & & & & & & & \\
\hline 02.01 .94 & 132 & & & & & & & 194 & & 124 & 82 & 186 & 171 & 125 & & & 93 & & & \\
\hline 08.01 .94 & 137 & & & & & & & 196 & & 129 & 86 & 197 & 178 & 127 & & & 100 & & & \\
\hline 14.01 .94 & 148 & & & & & & & 203 & & 128 & 86 & 210 & 189 & 135 & & & 106 & & & \\
\hline 21.01 .94 & 150 & & & & & & & 201 & & 126 & 86 & 213 & 194 & 136 & & & 107 & & & \\
\hline 28.01 .94 & 146 & & & & & & & 206 & & 126 & 88 & 213 & 180 & 130 & & & 108 & & & \\
\hline 04.02 .94 & 152 & & & & & & & 213 & & 128 & 90 & 209 & 182 & 143 & & & 112 & & & \\
\hline 07.02.94 & 152 & & & & & & & 209 & & 127 & 90 & 213 & 180 & 141 & & & 109 & & & \\
\hline 06.11 .98 & & & & & & & & & & & & & & & & & & & & 176 \\
\hline 09.11 .98 & & & & & & & & & & & & & & & & & & & & 189 \\
\hline 10.11 .98 & & & & & & & & & & & & & & & & & & & 161 & \\
\hline 16.11 .98 & & & & & & & 110 & & & & & & & & & & & & & \\
\hline 18.11 .98 & & & & & & & & & & & & & & & & & & & & \\
\hline 21.11 .98 & & & 114 & & & & 110 & & & & & & & & & 145 & & & & \\
\hline 23.11 .98 & & & & & & & & & 117 & & & & & & & & & & & \\
\hline 24.11 .98 & & & & & & & 98 & & & & & & & & & & & & & \\
\hline 25.11 .98 & & & & & & & & & 124 & & & & & & & & & & & \\
\hline 26.11 .98 & & & & & & & 91 & & & & & & & & & & & & & \\
\hline 29.11 .98 & & & 115 & & & & 88 & & & & & & & & & 138 & & & & \\
\hline 05.12 .98 & & & & & & & 97 & & & & & & & & & 128 & & & & \\
\hline 06.12 .98 & & & & & & 114 & & & & & & & & & & & & & & \\
\hline 09.12 .98 & & & & & & 121 & 102 & & & & & & & & & 128 & & & & \\
\hline 12.12 .98 & & & & & & & & & & & & & & & & 132 & & & & \\
\hline 13.12 .98 & & & & & & 117 & 109 & & & & & & & & & & & & & \\
\hline 15.12 .98 & & & & 110 & 164 & & & & & & & & & & & & & & & \\
\hline 16.12 .98 & & & & & & 106 & & & & & & & & & & & & & & \\
\hline 18.12 .98 & & & & 112 & 168 & 107 & 106 & & & & & & & & & 135 & & & & \\
\hline 21.12.98 & & & & 113 & 176 & 110 & 110 & & & & & & & & & 134 & & & & \\
\hline 26.12.98 & & & & 117 & 180 & 108 & 112 & & & & & & & & & 135 & & & & \\
\hline 30.12 .98 & & & & 119 & 175 & 108 & 114 & & & & & & & & & 138 & & & & \\
\hline 04.01.99 & & & & 119 & 185 & 109 & 118 & & & & & & & & & 142 & & & & \\
\hline 11.01 .99 & & & & 110 & 189 & 112 & 114 & & & & & & & & & 138 & & & & \\
\hline 18.01 .99 & & & & 111 & 186 & 116 & 107 & & & & & & & & & 142 & & & & \\
\hline
\end{tabular}




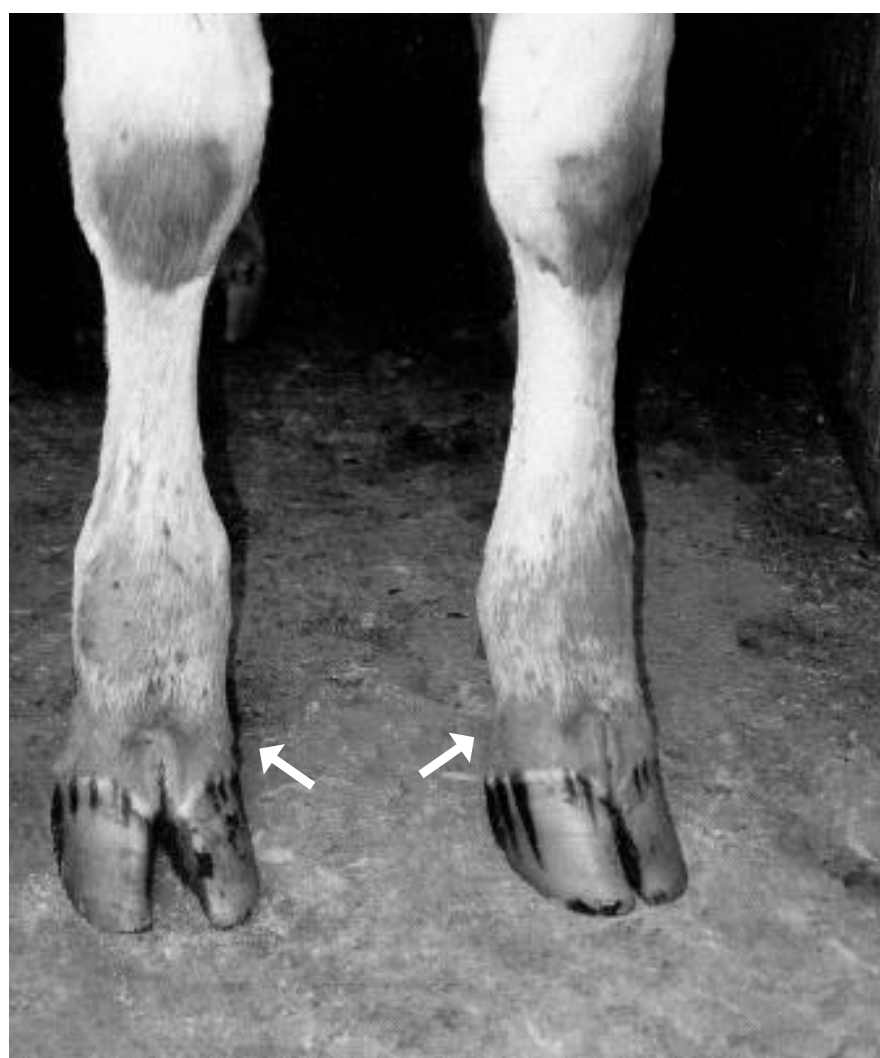

Fig. 8. Hiperemia e alopecia da extremidade distal dos membros anteriores (Bovino 181), na intoxicação experimental pelas favas de Stryphnodendron obovatum.

dade da urina dos bovinos por eles estudados esteve diminuída.

Segundo Kingsbury (1964), as saponinas contidas em algumas plantas podem causar desde irritação na parede do trato digestivo até uma severa gastrenterite. $O$ princípio tóxico das favas de $S$. obovatum ainda não foi identificado, porém Tursch et al. (1963) isolaram saponinas das favas de $S$. coriaceum.

Esse estudo sugere que as favas de $S$. obovatum provocam irritação no trato digestivo dos bovinos. A literatura consultada nos mostra que tanto $S$. obovatum, quanto $S$. coriaceum e até $S$. barbatimao produzem sintomas digestivos semelhantes, o que nos leva a crer que talvez saponinas sejam responsáveis ou pelo menos participem da patogenia também da intoxicação por S. obovatum.

Agradecimentos.- Ao Médico Veterinário Jaime Bomdespacho, Empaer/MT, e à Prof ${ }^{\mathrm{a}}$ Laura Maria Alvarez de Figueiredo, Unesp-Botucatu, SP, pela ajuda na coleta das favas, à Prof ${ }^{a}$ Elêna Maria Occhioni, UFRJ, pela identificação botânica, e aos técnicos João Luiz Bastos, Wilson Cabral da Fonseca e José Carlos Baeta, pelo apoio nos experimentos e trabalhos laboratoriais.

\section{REFERÊNCIAS}

Andrade S.O. \& Mattos J.R. 1968. Contribuição ao estudo de plantas tóxicas no Estado de São Paulo. Arqs Inst. Biológico, São Paulo, 122:39-41.

Baumgartner W. 1983. Klauentierpraxis. 5. Untersuchung des Pansensafts. Tierärztl. Umschau 38:558-561.

Camargo W. 1965. Intoxicação em bovinos por "barbatimão" (Stryphnodendron obovatum Benth., fam. Leguminosae). Arqs Inst. Biológico, São Paulo, 31(1):711.

Dirksen G. 1981. Indigestiones in el Bovino. Schnetztor-Verlag, Konstanz, Alemanha. 76p.

Dirksen G. 1969. Ist die Methylenblauprobe als Schnelltest fuir die klinische Pansensaftuntersuchung geeignet? Dtsch. Tierärztl. Wschr. 76:305-309.

Döbereiner J. \& Canella C.F.C. 1956. Intoxicação experimental pela fava do "barbatimão" (Stryphnodendron coriaceum Bth.) em bovinos. Bolm Soc. Bras. Med. Vet. 24:49-68.

Kingsbury J.M. 1964. Poisonous Plants of the Unites States and Canada. Prentice-Hall, New York, p.32-33.

Nichols R.E. \& Penn K.E. 1958. Simple methods for the detection of unfavorable changes in ruminal ingesta. J. Am. Vet. Med. Assoc. 133:275-277.

Occhioni E.M. 1990. Considerações taxonômicas no gênero Stryphnodendron Mart. (Leguminosae Mimosoideae) e distribuição geográfica das espécies. Acta Bot. Bras. 4(2):153-158.

Pereira C.A., Pessoa J.M. \& Santos H.L. 1989. Intoxicação experimental em bovinos pela fava do "barbatimão" (Stryphnodendron barbatimao Mart.). I. Sinais clínicos. Arq. Bras. Med. Vet. Zootec. 41(5):389-403.

Pereira C.A., Pessoa J.M. \& Santos H.L. 1989. Intoxicação experimental em bovinos pela fava do "barbatimão" (Stryphnodendron barbatimao Mart.). II. Aspectos anatomo-histopatológicos. Arq. Bras. Med. Vet. Zootec. 41(5):405-413.

Pereira C.A., Pessoa J.M. \& Santos H.L. 1989. Intoxicação experimental em bovinos pela fava do "barbatimão" (Stryphnodendron barbatimao Mart.). III. Dados laboratoriais. Arq. Bras. Med. Vet. Zootec. 41(5):405-413.

Slanina L. \& Rossow N. 1964. Zur speziellen Diagnostik einiger Erkrankungen des Vormagen-Labmagen-Komplexes. Monatsh. Veterinärmed. 19:282-291.

Tokarnia C.H., Brito M.F., Driemeier D., Costa J.B.D. \& Camargo A.J.R. 1998. Aborto em vacas na intoxicação experimental porStryphnodendron obovatum (Leg. Mimosoideae). Pesq. Vet. Bras. 18(1):35-38.

Tokarnia C.H., Döbereiner J. \& Peixoto P.V. 2000. Plantas Tóxicas do Brasil. Editora Helianthus, Rio de Janeiro. 320p.

Tokarnia C.H., Peixoto P.V., Gava A. \& Döbereiner J. 1991. Intoxicação experimental por Stryphnodendron coriaceum (Leg. Mimosoideae). Pesq. Vet. Bras. 11(1/2):25-29.

Tursch B., Tursch E., Harrison I.T., Silva G.B.C.T.C.B., Monteiro H.J., Gilbert B., Mors W.B. \& Djerassi C. 1963. Terpeinoides LIII. Demonstration of ring conformational changes in triterpenes of the $\beta$-amyrin class isolated from Stryphnodendron coriaceum. J. Org. Chemistry 28:2390-2394. 